\title{
GCSTLPP: Face Recognition using Gabor Center-Symmetric Tensor Locality Preservative Projection Approach in Video
}

\author{
Rajeshwari.J \\ DayanandaSagar College of Engineering, Information Science and Engineering Department, Bangalore, India \\ Email: raji_jkl@yahoo.co.in \\ K. Karibasappa \\ Oxford College of Engineering, Bangalore, India \\ Email: k_karibasappa@hotmail.com \\ Gopalkrishna M.T \\ K.S. School of Engineering and Management, Computer Science and Engineering Department, Bangalore, India \\ Email: gopalmtm@gmail.com
}

\begin{abstract}
Face Recognition has become the challenging and interesting research topic in the last few years. The aim is to design a robust Face Recognition System under different environmental conditions like illumination, pose and occlusion. These are the three major challenges in Face Recognition which may hinder the Face Recognition system. By combining the three successful representations such as Gabor filters, CS-LBP and TLPP better performance can be achieved as compared to just considering them individually. CS-LBP is used for describing interest regions which have good tolerance to illumination and computational efficiency and TLPP is used to take the data directly in the form of tensors as input. Since the number of the combined feature sets are more only a few feature sets is selected to be trained by the Support Vector Machine classifier. A number of experiments are conducted using YouTube celebrity, McGill Face dataset and as well as the own collected sequence under different conditions such as illumination variations, different poses, occlusion including indoor and outdoor scenes. This approach provides better results compared to traditional approaches.
\end{abstract}

Index Terms-Face Recognition, Gabor Filter, Center Symmetric Local binary pattern (LBP), Tensor locality preserving projections (TLPP).

\section{INTRODUCTION}

Face Recognition has gained its importance in the biometric field for understanding and analysis of images during past few years. Another biometric method such as palm print, finger print and iris recognition has better recognition rates, but it requires observance during the recognition process. Face Recognition can be performed with a lesser degree of compliance. The Face Recognition provides the high degree of recognition accuracy, but accuracy decreases when there is a variation in illumination, pose and occlusion and there will be crowd environment in the video surveillance [1]. These challenging issues may change the appearance of the face image and hinder the correct recognition process. The major applications of Face Recognition include Face Identification, Access Control, Security, Image Database Investigation, General Identity Verification, and Surveillance [2]. Some of the holistic approaches for Face Recognition are based on gray scale images, and converting from gray scale image may lead to loss of information. So we propose Face Recognition system based on color images since they contain some additional biometric information. Most of the recognition systems use one type of feature. For Face Recognition, no single feature modality is rich enough to capture all the classification information present in the image. Finding and combining complementary feature sets has now become an active research in pattern recognition. Xiaoyang Tan et al. [3] proposed fusion of Gabor and LBP which provided efficient Face Recognition under uncontrolled lighting. Rajeshwari.J et al. [4] proposed fusion of Gabor and LPP which provided good results for occluded and non-occluded pixels. Gabor filters are reasonable models of the visual processing in visual cortex and are used to describe the local appearance of the human face [5]. Local Binary Patterns was first proposed by Ojala et al. [6] which efficiently summarizes the local structure of the images. Srinivasa Reddy et.al [7] obtained the texture features using Local Ternary Pattern(LTP) for recognition of the face. The centersymmetric local binary pattern(CS-LBP) [8] was proposed which has good tolerance to illumination changes and computational efficiency. Information fusion for visual recognition can occur either at feature level or at the decision level [9]. In feature-level methods, a single feature set is formed by combining several feature sets into single feature set that is used in the classifier, 
whereas, at the decision level several classifiers are combined to a strong classifier [10]. In the area of Face recognition, multiple classes of descriptors can be fused and for fine distinctions. In this paper, both local feature extraction methods such as Gabor filter, CS-LBP and holistic feature extraction method such as Tensor based TLPP are used for extracting the features. All the three features set becomes the promising candidate for the fusion. In Local Binary Pattern (LBP), texture descriptor has not been used for describing interest regions. To overcome this problem, CS-LBP is used for describing the interest regions. TLPP is used so that the input is efficiently characterized.

\section{PROPOSED APPROACH}

This paper describes Face Recognition framework that is capable of achieving higher recognition rate in video sequences. The proposed block diagram is shown in Fig 1. In the first step of our proposed method, local features of the face region are obtained by convoluting the Gabor filters with the face image and face image is divided into the small regions by using the CS-LBP operator. The histogram is then finally generated for each of the regions and a global histogram is obtained and finally, features of both the methods are concatenated with TLPP Features. TLPP features mainly consider data as tensors of arbitrary order as input. Since the feature sets are more only first few feature sets are selected and given to Nonlinear Support Vector Machine classifier which is used for the training and classification.

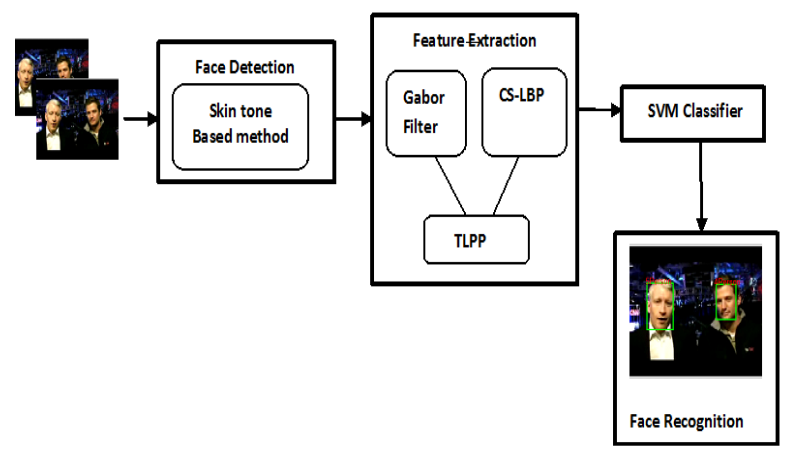

Fig.1. Block Diagram of the Proposed Approach

\section{A. Fusing the Features of Gabor filter and Centre- Symmetric LBP operator}

According to the literature survey, it's clear that high recognition rate is still a challenging task in different conditions. With this motivation, in the present work we have combined the features of Gabor filter, CentricSymmetric LBP and TLPP approach. Gabor filter is used for obtaining the localized frequency information. Gabor filters provide excellent spatial and frequency information. Gabor filters, CS-LBP and TLPP combined together to provide a better performance than individually. They are complimentary in the sense that Gabor filters with different frequencies and orientation are used for accessing the useful features of the image and CS-LBP operator has very good tolerance to illumination and computational efficiency and is used for describing the interest regions. TLPP is also used for extracting the features which use the multi-linear approach. Since the number of fused features is more, only first few features are selected. The stages of processing are shown in Fig 2.

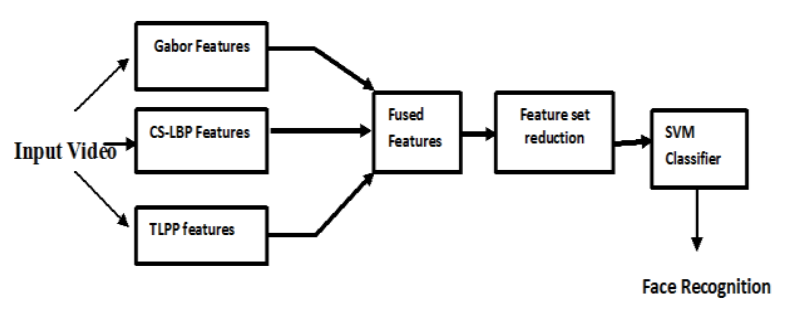

Fig.2. Stages of processing for Face Recognition system

Gabor feature extractor is used to find out the salient feature properties such as spatial localization, orientation selectivity and spatial frequency selectivity [11]. Gabor wavelets are suitable for image decomposition and representation for the derivation of local and discriminant features. Gabor wavelets are used for image analysis because of biological relevance and computational properties. Gabor features of an image are obtained by convolving the image with the Gabor filter of different scales and orientations. Let $\mathrm{f}(\mathrm{x}, \mathrm{y})$ be the face image, its convolution with the Gabor filter $\Psi_{\mu, v}(\mathrm{Z})$ is defined in Eq. "(1)"

$$
G_{\Psi, f}(\mathrm{x}, \mathrm{y}, \mu, \mathrm{v})=\mathrm{f}(\mathrm{x}, \mathrm{y})^{*} \Psi_{\mu, v}(\mathrm{Z})
$$

Two scales and two orientations are used. CenterSymmetric Local Binary Pattern (CS-LBP) combines the strength of both the SIFT and the LBP operator. The texture descriptor used in region description is the CSLBP operator [12]. The gradient feature in SIFT method is used in the CS-LBP operator which has simpler description than the SIFT method. It is most robust to illumination changes. The LBP operator produces the long histograms which are very difficult to use in the region descriptor.

The neighborhood of 8 pixels is shown in Fig 3. CSLBP operator produces 16 binary patterns for 8 neighbors because it does not compare with the center pixel but with the opposite pixels whereas the LBP produces the 256 different binary patterns which are given as follows:

$\mathrm{LBP}=\mathrm{s}(\mathrm{n} 0-\mathrm{nc}) 2^{0}+\mathrm{s}(\mathrm{n} 1-\mathrm{nc}) 2^{1}+\mathrm{s}(\mathrm{n} 2-\mathrm{nc}) 2^{2}+\mathrm{s}(\mathrm{n} 3-$ $n c) 2^{3}+\mathrm{s}(\mathrm{n} 4-\mathrm{nc}) 2^{4}+\mathrm{s}(\mathrm{n} 5-\mathrm{nc}) 2^{5}+\mathrm{s}(\mathrm{n} 6-\mathrm{nc}) 2^{6}+\mathrm{s}(\mathrm{n} 7$ $-\mathrm{nc}) 2^{7}$

$\mathrm{CS}-\mathrm{LBP}=\mathrm{s}(\mathrm{n} 0-\mathrm{n} 4) 2^{0}+\mathrm{s}(\mathrm{n} 1-\mathrm{n} 5) 2^{1}+\mathrm{s}(\mathrm{n} 2-\mathrm{n} 6) 2^{2}+$ $\mathrm{s}(\mathrm{n} 3-\mathrm{n} 7) 2^{3}[23]$

Robustness of the image regions is obtained by thresholding the gray level difference with the smaller value of $\mathrm{T}$. The value of the threshold $\mathrm{T}$ is actually set to 
0.01. The advantage of the CS-LBP operator over the LBP is it captures better gradient information and dimensionality reduction.

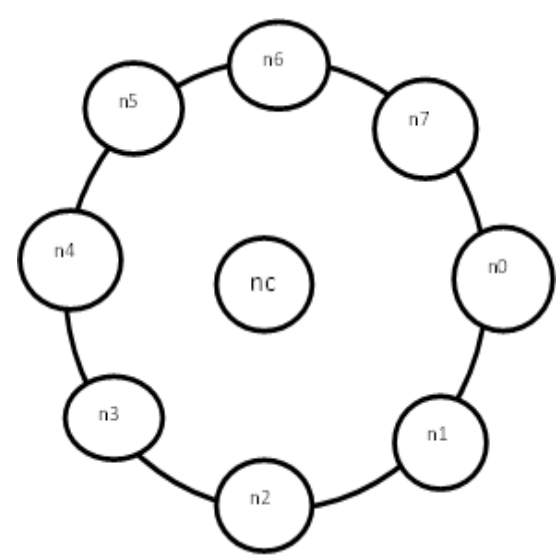

Fig.3. Neighborhood of 8 pixels

Tensor Locality preservative projection: LPP treats the object as vectored format. Image matrices have to be converted into the vectors which lead [13] to the high dimensional data but the tensor based algorithms directly treat the data as tensor and avoid problems of treating the data as vectors. Tensor LPP is the logical extension of the LPP in multi-linear mode. Tensor-LPP considers the data as tensors of arbitrary order as input and also allows the relationship between the dimensions of tensor representation to be efficiently characterized [14]. Given as an $\mathrm{N}$ data points $A_{1}, A_{2}, \ldots A_{n}$ from an unknown manifold $\mathrm{M}$ embedded in tensor space R1. In order to preserve the local topological structure of $M$ and effectively capture the intrinsic geometry, TLPP finds the $\mathrm{K}$ optimal projections. We construct a neighbourhood graph $\mathrm{G}$ to represent the local geometric of $\mathrm{M}$. The affinity matrix $\mathrm{S}=\left[S_{i, j}\right]_{n x n}$ is defined based on the heat kernel as:

$$
\begin{gathered}
s_{i, j}=\exp \left(-\left\|A_{i}-A\right\|_{f}^{2} / \mathrm{t}\right) \text { if } A_{i} \in \mathrm{O}\left(\mathrm{k}, A_{i}\right) \text { or } A_{j} \in \mathrm{O} \\
\left(\mathrm{k}, A_{j}\right)
\end{gathered}
$$

Where $\mathrm{O}\left(\mathrm{K}, \mathrm{A}_{\mathrm{i}}\right)$ denotes the $\mathrm{K}$ nearest points of $\mathrm{A}_{\mathrm{i}}$ and $\mathrm{t}$ is positive constant.

\section{B. SVM Classifier}

SVM classifier is used for binary classification which separates the classes with maximum margin and selects one particular solution. SVM learning algorithm analyzes the data and recognizes the patterns from a given set of training examples and marks it with one of the two categories. SVM training builds a model that assigns new features to one of the two categories. Given with set of $n$ points(vectors) $x_{1}, x_{2} \ldots x_{n}$ and $x_{i}$ is a length of $\mathrm{m}$ and each belongs to one of the two classes either by +1 or -1 . If the training set is $\left(x_{1}, y_{1}\right),\left(x_{2}, y_{2}\right) \ldots\left(x_{n}, y_{n}\right)$ for all i $x_{i} \in$ $\mathrm{Rm}$ and $y_{i} \in+1,-1$ classifier is learned $\mathrm{f}(\mathrm{x})=\operatorname{sign}(\mathrm{w} . \mathrm{x}$ + b) such that

$$
f(x)=\left\{\begin{array}{r}
>0, \text { if } y_{i}=+1 \\
0, \text { if } y_{i}=-1
\end{array}\right.
$$

Where $y_{i}=+1$ represents the face and $y_{i}=-1$ represents the non-face.

SVM will minimize the similarity between the classification hyper plane and the subspace of the solutions in class 1 . With new dimension, it examines for the linear optimal searching hyper plane i.e. decision boundary separating the samples of one class from another. To find the optimal separating hyper plane SVM aims to maximize the margin, as given below.

$$
\begin{gathered}
\text { Maximize }=2 /\|\mathrm{w}\| \\
f(x)=\left\{\begin{array}{l}
y_{i}=+1, \text { if } w \cdot x+b>0 \\
y_{i}=-1, \text { if } w \cdot x+b<0
\end{array}\right.
\end{gathered}
$$

\section{EXPERIMENTS}

\section{A. Database}

Two standard datasets i.e. YouTube Celebrity [15], McGill Face [16] are used to evaluate the performance of the GCSTLPP as shown in Fig 4. There are only a few face occurrences in the standard dataset, so we have collected our own video sequences with increased people count and different variations of environmental conditions both indoor and outdoor. We have considered totally 16 video sequences and each video consists of person ranging from one to ten. We have tested our proposed method on 20 frames of each video. So totally we have 780 faces to be recognized from all the video sequences.

\section{B. Comparative Study and Experimental Results}

PCA [17], LPP [18], TLPP and OLPP [19] are holistic feature extraction methods and their performance depends on the training set which is sensitive to illumination and pose but the local feature extraction methods such as Gabor [20] and LBP [21] [22] are more robust to environmental conditions. But, LBP has not been used for describing the interesting regions. CS-LBP overcomes this problem with computational efficiency. To avoid losing the global information we have used TLPP which takes the data directly in the form of tensors. Thus fused features of Gabor, CS-LBP and TLPP have been used so that our proposed method GCSTLPP gives good tolerance to illumination changes, pose and occlusion. Figure 5 and 6 show successively Face Recognition of standard YouTube Celebrity, McGill Face and own dataset respectively. In Fig 5 and 6, the first column shows input video frames, the second column shows results obtained by applying skin tone based method and the third column shows the result obtained for proposed method. In Fig 5, the result (c), (f) and (i) show that the efficient result is obtained for the proposed method in YouTube celebrity dataset for video recorded under bright illumination, under different pose variations and during the night. In Fig 5, the result (1) and (o) show 
the efficient result obtained for the proposed method in McGill Face dataset for video recorded with a person wearing spectacles and non-uniform background. In Fig 6 , result (c), (f), (i), (l) and (o) shows the efficient result obtained for the proposed method in own dataset under indoor area, outdoor plus during sunset condition, indoor plus non-uniform background, with twins and crowded area. In Fig 7 shows comparative results of proposed method GCSTLPP for Face Recognition in comparison with the existing approaches. Our proposed method gives the good recognition rate when compared to other methods, since we are extracting both local and global features without losing any information as shown in Table 1.

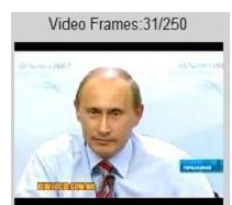

(a)

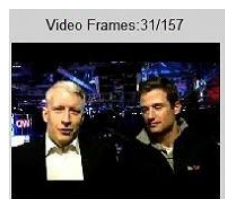

(b)

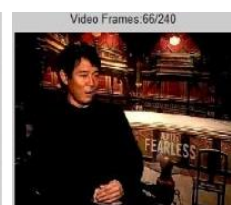

(c)

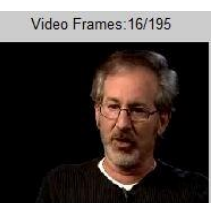

(d)

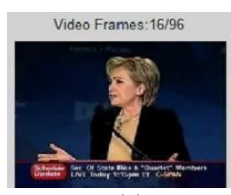

(e)

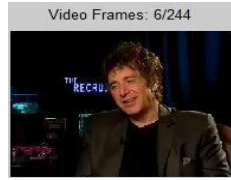

(f)

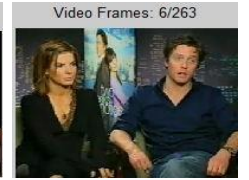

(g)

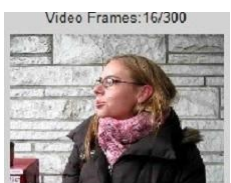

(h)

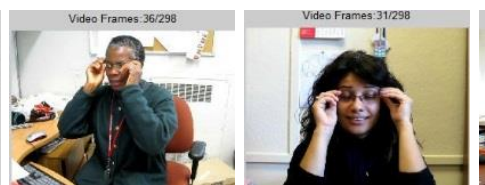

(i) (j)

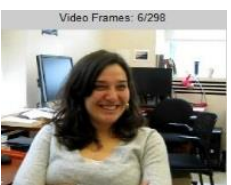

(k)

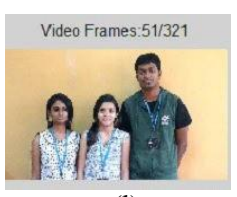

(1)

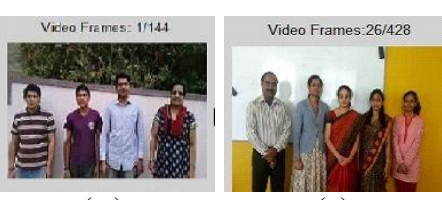

(m) (n)

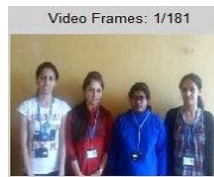

(o)

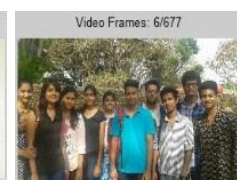

(p)

Fig.4. YouTube Celebrity Dataset:(a, b, c, d, e, f, g), McGill Face Dataset:(h, i, j, k), Own Dataset:(1, m, n, o, p)

Table 1. Recognition rate

\begin{tabular}{|c|c|c|c|c|c|c|c|}
\hline Dataset & PCA & LBP & LPP & TLPP & OLPP & Gabor & $\begin{array}{c}\text { Proposed } \\
\text { Approach }\end{array}$ \\
\hline YouTube Celebrity & 90.5 & 100 & 89.94 & 87.77 & 80.44 & 91.62 & 98.88 \\
\hline McGill Face & 55.0 & 63.29 & 83.33 & 82.5 & 78.75 & 87.5 & 85.0 \\
\hline Own Video & 71.4 & 85.71 & 76.67 & 81.46 & 80.0 & 81.13 & 90.53 \\
\hline & 74.26 & 86.75 & 80.6 & 83.1 & 80.0 & 83.0 & 91.94 \\
\hline
\end{tabular}

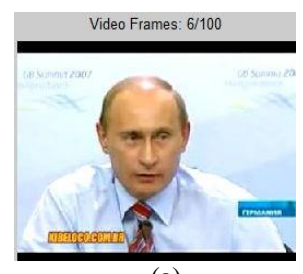

(a)

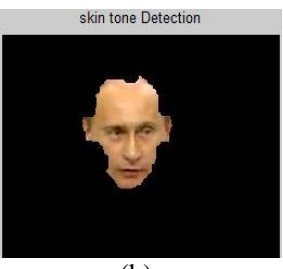

(b)

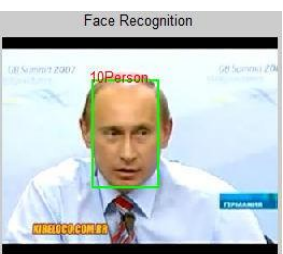

(c) 


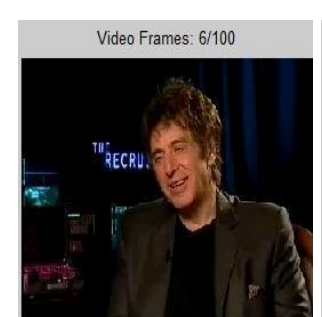

(d)

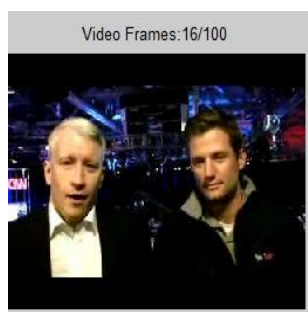

(g)

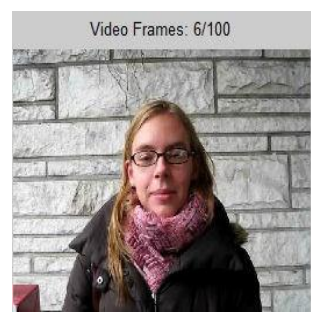

(j)

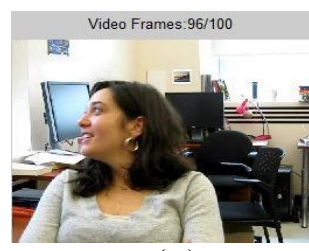

(m)

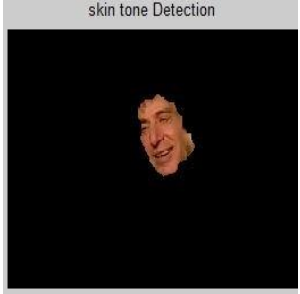

(e)

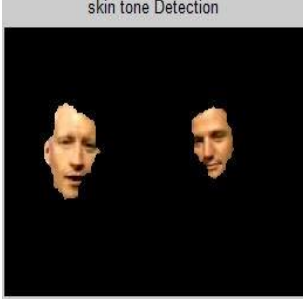

(h)

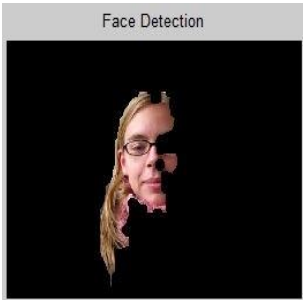

(k)

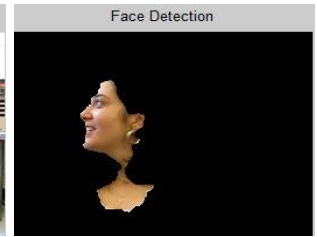

(n)

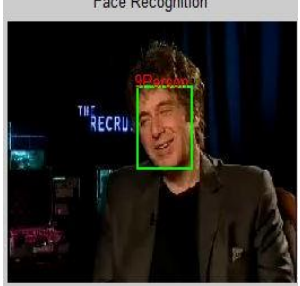

(f)

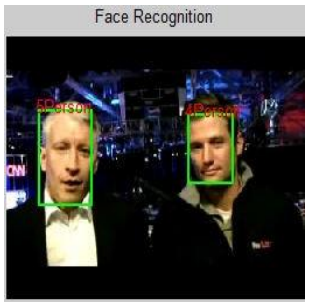

(i)

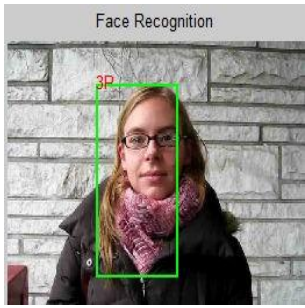

(1)

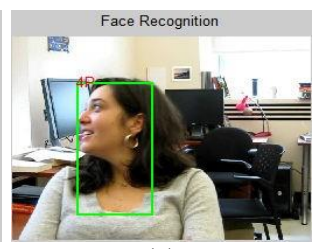

(o)

Fig.5. Results of the proposed method on YouTube Celebrity Face Dataset: Bright Illumination (a, b, c), Pose Variation (d, e, f), Night (g, h, i), McGill face Dataset: Occlusion (j, k, l), Non-Uniform background (m, n, o).

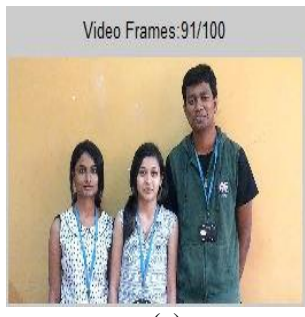

(a)

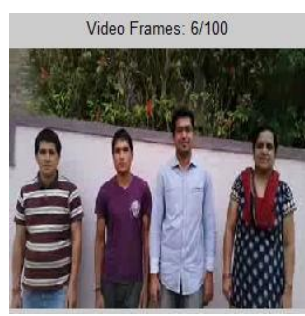

(d)

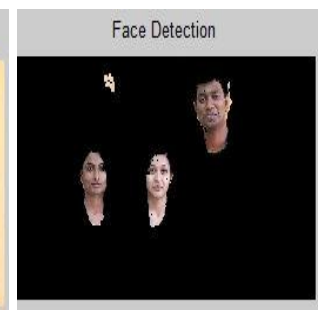

(b)

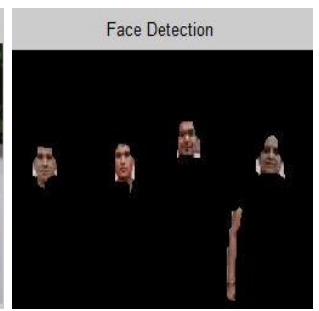

(e)

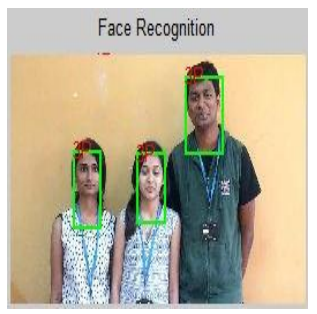

(c)

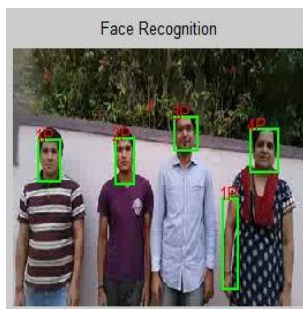

(f) 


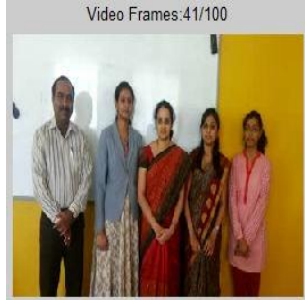

(g)

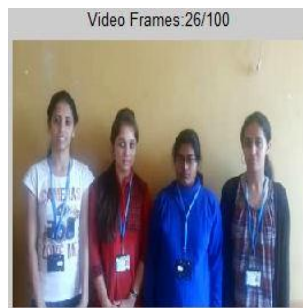

(j)

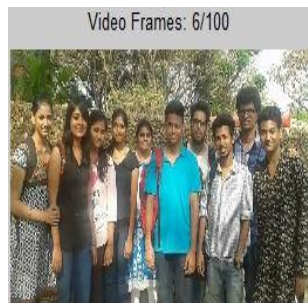

(m)

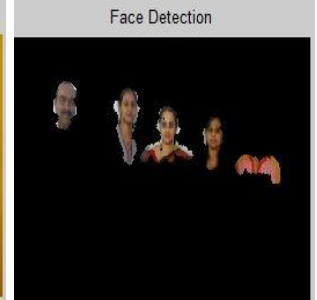

(h)

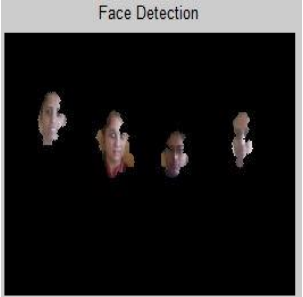

(k)

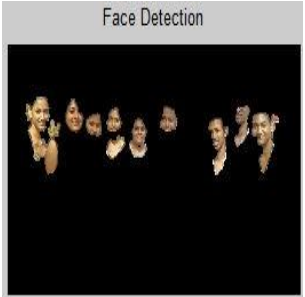

(n)

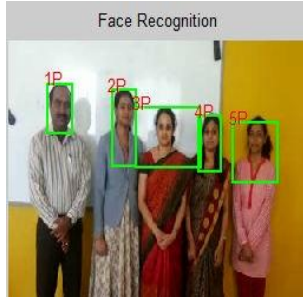

(i)

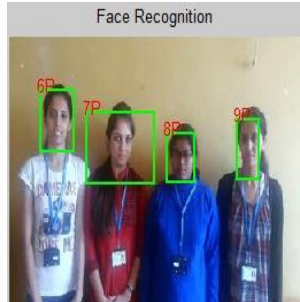

(1)

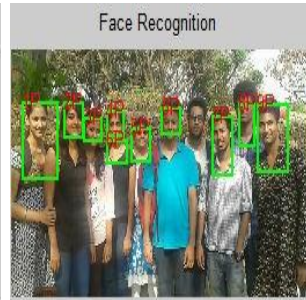

(o)

Fig.6. Results of Proposed method on Own Face Dataset: Indoor Scene (a, b, c), Outdoor sunset (d, e, f), Indoor Non-Uniform background (g, h, i), twins $(\mathrm{j}, \mathrm{k}, \mathrm{l})$, Crowded scene $(\mathrm{m}, \mathrm{n}, \mathrm{o})$

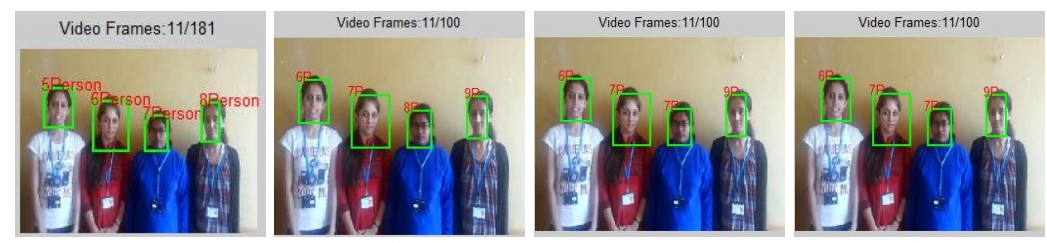

(a) (c) (d)

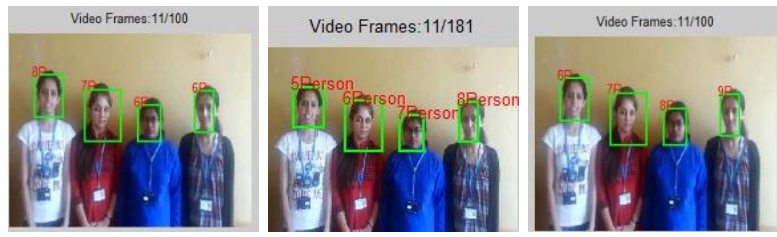

(e)

(f)

(g)

Fig.7. Comparative results of PCA(a), LBP(b), LPP(c), TLPP(d), OLPP(e), Gabor(f) Proposed method(g)

\section{CONCLUSION}

In this paper, a novel method for Face Recognition is implemented on both standard and own datasets. In contrast with the traditional approaches for Face Recognition, a new approach GCSTLPP is proposed. A fused feature set of Gabor, CSLBP and TLPP gives better performance rather than considering individually. Each of the feature sets has its own significance such as Gabor filter has excellent spatial and frequency information, CSLBP operator captures better gradient information and dimensionality reduction and TLPP treats data directly as tensors and avoids problems of treating the data as vectors. The experiments done on this proposed method provides good recognition rate compared to other existing approaches.

\section{REFERENCES}

[1] Rajeshwari J, Veena H L , Dr. K. Karibasappa, Video Based Face Recognition Using Ga- bor Features and LBP under Varying Illumination or Pose, International Journal of Innovative Research in Computer and Communication Engineering, Vol. 3, Issue 6, June 2015

[2] Divyarajsinh N. Parmar, Brijesh B. Mehta, Face Recognition Methods Applications, Int.J. Computer Technology Applications, Vol 4 (1),84-86, IJCTA — JanFeb 2013

[3] Xiaoyang Tan and Bill Triggs, Fusing Gabor and LBP Feature Sets for Kernel-Based Face Recognition, AMFG 
2007, LNCS 4778, pp. 235-249, 2007. Springer-Verlag Berlin Heidelberg 2007

[4] Rajeshwari J, ReeniyaBoopaiah, K.Karibasappa, ,Video Based Face Recognition with Gabor Features and Locality Preserving Projections under Varying Partial Occlusion, International Journal of Innovative Research in Computer and Communication Engineering, Vol. 3, Issue 6, June 2015

[5] Lee T.S, Image Representation Using 2D Gabor Wavelets, IEEE Trans. Pattern Analysis and Machine Intelligence, Vol 18, No. 10, pp.959-971, Oct. 1996.

[6] Ojala T, Pietikainen M, and Harwood D, A comparative study of texture measures with classification based on feature distributions, Pattern Recognition, Vol 29, No. 1, pp.51-59, January 1996.

[7] K. Srinivasa Reddy, V. Vijaya Kumar, B. Eswara Reddy, Face Recognition Based on Texture Features using Local Ternary Pattern, I.J. Image, Graphics and Signal Processing, 2015, 10, 37-46, Published Online September 2015, in MECS.

[8] DeepthyBoban, Cinita Mary Mathew, Detecting Surgically Altered Face Images Using CS-LBP and Genetic Algorithm, International Journal of Advanced Research in Com- puter Science and Software Engineering Volume 4, Issue 8, August 2014.

[9] Jain A, Nandakumar, K, Ross A, Score normalization in multimodal biometric systems Pattern Recognition 38(12), 2270-2285 (2005).

[10] Kittler J, Hatef, M., Duin, R.P, Matas, J, on combining classifiers, IEEE TPAMI 20(3), 226-239 (1998)

[11] Quan-You Zhao, Bao-Chang Pan, Jian-Jia Pan, Yuan-Yan Tang, Facial Expression Recognition Based On Fusion Of Gabor And LBP Features, Proceedings of the 2008 International Conference on Wavelet Analysis and Pattern Recognition, Hong Kong, 30- 31 Aug. 2008

[12] Loris Nanni, Alessandra Lumini, Random interest regions for object recognition based on texture descriptors and bag of features, ARTICLE in Expert Systems with Applications January 2012

[13] DazhaoZheng, XiufengDu, Limin Cui, Tensor Locality Preserving Projections for Face Recognition, Systems Man and Cybernetics (SMC), IEEE International Conference, 2010

[14] M.T.Gopalakrishna,M, Ravishankar,D, R.Rameshbabu, Ten-LoPP: Tensor Locality Pre- serving Projections Approach for Moving Object Detection and Tracking, The 9th Inter- national Conference on Computing and Information Technology (IC2IT2013) Advances in Intelligent Systems and Computing Volume 209, 2013, pp 291-300

[15] M. Kim, S. Kumar, V. Pavlovic, and H. Rowley, Face Tracking and Recognition with Visual Constraints in RealWorld Videos, Proc. 2008 IEEE Conference on Computer Vision and Pattern Recognition, pp. 18, 2008

[16] M. Demirkus, J. J. Clark and T. Arbel, Robust SemiAutomatic Head Pose Labeling for Real-World Face Video Sequences, Multimedia Tools and Applications, January 2013

[17] Turk M, Pentland A: Eigenfaces for Recognition. Cogn.Neurosci. 3(1), 71-86 (1991)

[18] Xiaofei He and ParthaNiyogi, Locality preserving projections, In Proc. of NIPS., Department of Computer Science, University of Chicago, Chicago,2003

[19] D.Cai, X.He and J.W.Han, Orthogonal laplacian faces for face recognition, IEEE Trans. on Image Procession, vol. 15 , no. $11,2006$.
[20] Liu C, Wechsler H, Gabor feature based classification using the enhanced fisher linear discriminant model for face recognition, IEEE Transactions on Image Processing, 11 (2002) 467-476.

[21] Ojala T, Pietikainen M, Maenpaa T, Multi-resolution grayscale and rotation invariant texture classification with local binary patterns, IEEE TPAMI 24(7), 971-987 (2002)

[22] Ahonen T, Hadid A, Pietikainen M, Face description with local binary patterns: Application to face recognition, IEEE TPAMI 28(12) (2006)

[23] Heikkila Marko, PietikainenMatti, SchmidCordelia, Description of interest regions with center-symmetric local binary patterns, 5th Indian Conference on Computer Vision, Graphics and Image Processing, ICVGIP 2006. Madurai, Inde, December, 2006. Lecture Notes in Computer Science volume 4338, pages 58-69. 2006.

\section{Authors' Profiles}

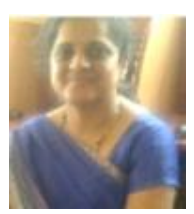

Rajeshwari J obtained her Bachelor's degree in Computer Science and Engineering from Gulbarga University in 2001 and M.Tech degree in Computer Network Engineering from VTU, India in 2007. Since 2010 she has been a Ph.D. student at Visvesvaraya Technological University, India. Currently, she is working as an Associate Professor in Information Science and Engineering department, Dayananda Sagar College of Engineering, Bangalore India. She has teaching experience of more than 13 years. Her research interest includes image and video processing, pattern recognition and Computer Networks. She has published more than 10 papers in International Journals, International conferences.

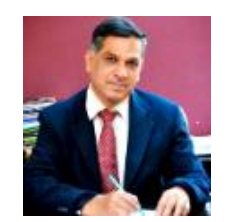

Dr.K. Karibasappa received B.E. (Electronics and Communication Engineering) degree from Malnad College of Engineering, Hassan, Karnataka, India in 1985, M.Tech degree from Jadavapur University India in 1998, and Ph.D. degree from Sambalpur University, India in 2004. He has more than 30 years of teaching experience. He is currently working as Principal of Oxford College of Engineering, Bangalore, India. He has published more than 50 papers in various International journals, International conferences and National conferences. His current research interests are Digital Image Processing, Machine Learning and perception, Artificial Intelligence, Data Mining, Knowledge Acquisition.

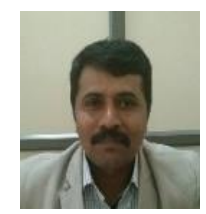

Dr. M. T Gopalakrishna received B.E degree (Computer Science \& Engineering) in 1998 from M. S Ramaiah Institute of Technology, India, M.Tech degree from Visvesvaraya Technological University, Karnataka, India and $\mathrm{PhD}$ from Visvesvaraya Technological University, Karnataka, India. He has more than 16 years of teaching experience. He is currently Professor in K.S School of Engineering, Bangalore, India. He has published more than 30 papers in various International journals, International conferences and National conferences. His current research is Pattern Recognition, Digital Image Processing \& Computer Vision 